\title{
On Spin Structures and Dirac Operators on the Noncommutative Torus
}

\author{
Mario Paschke \\ Max-Planck-Institut für Mathematik in den Naturwissenschaften \\ Inselstr. 22, 04103 Leipzig, Germany \\ Andrzej Sitarz ${ }^{* \dagger}$ \\ Institute of Physics, Jagiellonian University \\ Reymonta 4, 30-059 Kraków, Poland \\ and \\ Mathematisches Institut, Heinrich-Heine-Universität \\ Universitätsstrasse 1, 40225 Düsseldorf, Germany
}

\begin{abstract}
We find and classify possible equivariant spin structures with Dirac operators on the noncommutative torus, proving that similarly as in the classical case the spectrum of the Dirac operator depends on the spin structure.
\end{abstract}

MSC 2000: 58B34, 46L87

Keywords: spectral geometry, noncommutative geometry

\section{Introduction}

Unlike classical differential geometry, in Noncommutative Geometry there seems to be no well-established, commonly accepted notion of spin structures over noncommutative manifolds. The notion of a real spectral triple [2], which provides

*Alexander von Humboldt Fellow

${ }^{\dagger}$ Partially supported by MNII Grant 115/E-343/SPB/6.PR UE/DIE 50/2005-2008 
a noncommutative counterpart of the spin manifold definition incorporates in the classical case both spin structure and the Dirac operator. In the known examples, it is a priori difficult to separate these two ingredients. On the other hand, one would expect that possible spectral triple constructions should - like in the classical case - depend on some possible choices of the reality structure $J$. The resulting Dirac operators might then have different spectra.

In the paper we investigate the simplest and most studied example of the twodimensional noncommutative torus. In the classical limit, it is well known that there are four different spin structure on the torus and the Dirac operators have different spectra [1]. We recover this result in the noncommutative case, showing that out of several a priori admissible reality structures only some admit reasonable Dirac operators.

For the details of the spectral triple axiomatics we refer the reader to [3]. In our approach we use equivariance under the global symmetry of the noncommutative manifolds as defined in [4, 5].

\section{Spectral triples on Noncommutative Torus}

\subsection{Equivariant representation and a real structure}

Let $U, V$ be unitary elements generating the algebra of polynomial functions on the noncommutative torus $\mathcal{A}\left(T_{\lambda}\right), \lambda$ a generic complex number, $|\lambda|=1$,

$$
U V=\lambda V U
$$

and $\delta_{1}, \delta_{2}$ be the basis of derivations acting on $\mathcal{A}\left(T_{\lambda}\right)$, which represent the Lie algebra type symmetry (denoted later by $\mathcal{L}$ ) of the noncommutative torus:

$$
\begin{aligned}
& \delta_{1} \triangleright U=U, \quad \delta_{2} \triangleright U=0, \\
& \delta_{1} \triangleright V=0, \quad \delta_{2} \triangleright V=V \text {. }
\end{aligned}
$$

We look for all possible equivariant representations with an equivariant real structure $J$ and, since the spectral triple for a two-dimensional torus should be even, for a $\mathbb{Z}_{2}$ grading $\gamma$. Note that in this case the Hilbert space must be graded and we need to find two equivariant representations $\pi_{1}, \pi_{2}$.

First, we assume that $\mathcal{V}$ is a vector space on which we have a well-defined star-representation $\rho$ of the derivations and that $e_{\mu, \nu}$ are their mutual eigenvectors:

$$
\rho\left(\delta_{1}\right) e_{\mu, \nu}=\mu e_{\mu, \nu}, \quad \rho\left(\delta_{2}\right) e_{\mu, \nu}=\nu e_{\mu, \nu}
$$


where $\mu, \nu \in \mathbb{R}$ are arbitrary numerical labels.

The equivariance condition, for $l \in \mathcal{L}$ and $a \in \mathcal{A}\left(T_{\lambda}\right), v \in \mathcal{V}$ :

$$
\rho(\ell) \pi(a) v=(\pi(\ell \triangleright a)+\pi(a)) v
$$

gives us the following result

$$
\pi(U) e_{\mu, \nu}=u_{\mu, \nu} e_{\mu+1, \nu}, \quad \pi(V) e_{\mu, \nu}=v_{\mu, \nu} e_{\mu, \nu+1} .
$$

Further, from the commutation relation (10) we get

$$
u_{\mu, \nu+1} v_{\mu, \nu}=\lambda u_{\mu, \nu} v_{\mu+1, \nu} .
$$

Since the eigenvectors $e_{\mu, \nu}$ can always be rescaled by a suitable phase we might choose,

$$
u_{\mu, \nu}=1, \quad v_{\mu, \nu}=\lambda^{-\mu}
$$

Therefore, the minimal irreducible equivariant representation would consists of a linear span of $e_{\mu+m, \nu+n}$ for all $m, n \in \mathbb{Z}$ :

$$
\mathcal{V}_{\mu, \nu}=\bigoplus_{m, n \in \mathbb{Z}} V_{\mu_{0}+m, \nu_{0}+n}
$$

where each $V_{\mu_{0}+m, \nu_{0}+n} \simeq \mathbb{C}$.

Note that we have obtained no restriction for the values of $\mu_{0}, \nu_{0}$ and, a priori, the values for each of the two representations might be chosen independently and be completely different. We shall label the two eigenspaces of the grading $\mathcal{V}^{+}$and $\mathcal{V}^{-}$and take $\mathcal{V}=\mathcal{V}_{+} \oplus \mathcal{V}_{-}$.

Next, we look for an antilinear unitary operator $J$ on $\mathcal{V}$, such that:

$$
J^{2}=-1, \quad J \gamma=-\gamma J
$$

The latter condition means that $J$ maps $\mathcal{V}_{ \pm}$onto $\mathcal{V}_{\mp}$. We use the equivariance condition for $J$, which, in the case of Lie-algebra symmetry, reads:

$$
-J \rho\left(\ell^{*}\right) v=\rho(\ell) J v, \quad \forall v \in \mathcal{V}, \ell \in \mathcal{L} .
$$

Writing it explicitly for the derivations $\delta_{1}, \delta_{2}$ :

$$
\begin{aligned}
-\left(\mu_{1}+m_{1}\right) J e_{\mu_{1}+m_{1}, \nu_{1}+n_{1}, \pm} & =\delta_{1}\left(J e_{\mu_{1}+m_{1}, \nu_{1}+n_{1}, \pm}\right) \\
-\left(\nu_{1}+n_{1}\right) J e_{\mu_{1}+m_{1}, \nu_{1}+n_{1}, \pm} & =\delta_{2}\left(J e_{\mu_{1}+m_{1}, \nu_{1}+n_{1}, \pm}\right) .
\end{aligned}
$$

Hence, we immediately get: 
Lemma 2.1. An equivariant $J: \mathcal{V} \rightarrow \mathcal{V}$ satisfying (8) exists only and only if the spectrum of the derivations $\delta_{1}, \delta_{2}$ on spaces $\mathcal{V}_{+}$is a symmetric image (with respect to $x \rightarrow-x$ ) of the spectrum on $\mathcal{V}_{-}$. Then:

$$
J e_{\mu, \nu, \pm}= \pm j(\mu, \nu) e_{-\mu,-\nu, \mp},
$$

where $j(\mu, \nu)$ is an arbitrary phase and $e_{\mu, \nu, \pm} \in \mathcal{V}_{ \pm}$.

Proof. The equation (11) is direct consequence of (10). Clearly, if $\mu$ is in the spectrum of $\delta_{1}$ on $\mathcal{V}_{+}$then $-\mu$ must be in the spectrum of $\delta_{1}$ on $\mathcal{V}_{-}$.

Next, by requiring that $J$ maps the algebra to its commutant, we have

Lemma 2.2. $J$ maps the algebra $\mathcal{A}\left(T_{\lambda}^{2}\right)$ to its commutant if and only if

$$
j(\mu, \nu)=e^{i \phi \mu+i \psi \nu+\theta} \lambda^{-\mu \nu},
$$

where $\phi, \psi$ and $\theta$ are arbitrary real numbers.

Proof. First, observe that $J$ is of the form $J=\Lambda J_{0}$, where $J_{0}$ is antilinear and $\Lambda$ is diagonal and unitary:

$$
J_{0} e_{\mu, \nu, \pm}= \pm e_{-\mu,-\nu, \mp}, \quad \Lambda e_{\mu, \nu, \pm}=j(-\mu,-\nu) e_{\mu, \nu, \pm} .
$$

Taking first $j(\mu, \nu)=\lambda^{-\mu \nu}$ we verify explicitly that such canonical $J_{c}$ maps the algebra to the commutant:

$$
\begin{aligned}
& U^{o} e_{\mu, \nu, \pm}=J_{c}^{*} U^{*} J_{c} e_{\mu, \nu, \pm}=\lambda^{-\nu} e_{\mu+1, \nu, \pm} \\
& V^{o} e_{\mu, \nu, \pm}=J_{c}^{*} V^{*} J_{c} e_{\mu, \nu, \pm}=e_{\mu, \nu+1, \pm}
\end{aligned}
$$

and it is easy to verify that $U^{o}, V^{o}$ are indeed in the commutant of $\mathcal{A}\left(T_{\lambda}\right)$.

Now, assume that there exists a different diagonal unitary $\Lambda^{\prime}$, such that $J^{\prime}=$ $\Lambda J_{0}$ maps the algebra to the commutant. Consider the map $W=J^{\prime} J_{c}$ :

$$
J^{\prime} J_{c} e_{\mu, \nu, \pm}=w_{ \pm}(\mu, \nu) e_{\mu, \nu, \pm}
$$

Clearly, $W=J^{\prime} J_{c}$ is unitary. Both $W^{*} U W$, and $W^{*} V W$ should commute with $U^{o}$ and $W^{o}$, for instance:

$$
\left[\left(J^{\prime} J_{c}\right)^{*} U\left(J^{\prime} J_{c}\right), J_{c}^{*} U J_{c}\right]=J_{c}^{*}\left[\left(J^{\prime}\right)^{*} U J^{\prime}, U\right] J_{c}=0
$$


since we assumed that $J^{\prime}$ also maps $\mathcal{A}\left(T_{\lambda}\right)$ to the commutant. Let us calculate $\left[W^{*} U W, V^{o}\right]:$

$$
\begin{aligned}
{\left[W^{*} U W, U^{o}\right] e_{\mu, \nu, \pm}=} & \lambda^{-\nu}\left(w_{ \pm}(\mu+1, \nu) w_{ \pm}(\mu+2, \nu)^{*}\right. \\
& \left.-w_{ \pm}(\mu, \nu) w_{ \pm}(\mu+1, \nu)^{*}\right) e_{\mu+2, \nu, \pm}
\end{aligned}
$$

Therefore, using the unitarity of $W$ :

$$
\left(w_{ \pm}(\mu+1, \nu)\right)^{2}=w_{ \pm}(\mu, \nu) w_{ \pm}(\mu+2, \mu),
$$

This recurrence relation has the following solution:

$$
w(\mu, \nu)=e^{i \mu \phi_{ \pm}} w_{ \pm}^{0}(\nu),
$$

where $\phi_{ \pm}$are arbitrary constants and $w_{ \pm}^{0}$ an arbitrary unitary function of $\nu$. Next:

$$
\begin{aligned}
{\left[W^{*} V W, V^{o}\right] e_{\mu, \nu, \pm}=} & =\lambda^{-\mu}\left(w_{ \pm}(\mu, \nu) w_{ \pm}(\mu, \nu+1)^{*}\right. \\
& \left.-w_{ \pm}(\mu, \nu+1) w_{ \pm}(\mu, \nu+2)^{*}\right) e_{\mu, \nu+2, \pm} .
\end{aligned}
$$

Using (15) this leads to:

$$
\left(w_{ \pm}^{0}(\nu+1)\right)^{2}=w_{ \pm}^{0}(\nu) w_{ \pm}^{0}(\nu+2),
$$

which yields the final general solution:

$$
w_{ \pm}(\mu, \nu)=e^{i \phi_{ \pm} \mu+i \psi_{ \pm} \nu+i \theta_{ \pm}}
$$

It is an easy exercise to see that the remaining commutators vanish as well.

The relation between $\phi_{+}$and $\phi_{-}$(and similarly for $\psi_{ \pm}, \theta_{ \pm}$) can be fixed using the requirement of unitarity of $J^{\prime}$ and the first relation (8). Hence, one gets that: $J^{\prime}=-W J$ must be:

$$
J^{\prime} e_{\mu, \nu, \pm}= \pm e^{ \pm(i \phi \mu+i \psi \nu+i \theta)} \lambda^{-\mu \nu} e_{-\mu,-\nu, \mp} .
$$

The constant $\theta$ is a global phase and can be fixed to $\theta=0$.

It is interesting to observe what the map $W$ is doing. Clearly $W^{*} \pi(a) W$ commutes with the commutant of $\mathcal{A}\left(T_{\lambda}\right)$ but is not in the chosen representation of $\mathcal{A}\left(T_{\lambda}\right)$. In fact, the conjugation by $W$ induces an automorphism of $\mathcal{A}\left(T_{\lambda}\right)$ but only when restricted to the single representation $\pi_{+}$or $\pi_{-}$:

$$
\begin{array}{ll}
W_{+}^{*} \pi_{+}(U) W_{+}=\pi_{+}\left(e^{-i \phi} U\right), & W_{-}^{*} \pi_{-}(U) W_{-}=\pi_{-}\left(e^{i \phi} U\right), \\
W_{+}^{*} \pi_{+}(V) W_{+}=\pi_{+}\left(e^{-i \psi} V\right), & W_{-}^{*} \pi_{-}(V) W_{-}=\pi_{-}\left(e^{i \psi} V\right),
\end{array}
$$

where $W_{ \pm}$denotes the respective diagonal components of $W$. 


\subsection{The equivariant Dirac operator}

We proceed now with the construction of an equivariant Dirac operator. Assume the existence of an equivariant graded linear operator, that is an operator, which commutes with derivations and anticommutes with $\gamma$. From this we infer that:

$$
D e_{\mu, \nu, \pm}=d_{\mu, \nu, \pm} e_{\mu, \nu, \mp}
$$

Therefore, $D$ intertwines vectors of the same eigenvalues of the derivations $\delta_{1}, \delta_{2}$ and in both subspaces such vectors must be present. On the other hand, from the action of $J$ we know that for each vector with eigenvalues $\mu, \nu$ in one space there exists one with eigenvalues $-\mu,-\nu$. Hence, we must have:

$$
\exists m, n \in \mathbb{Z}: \mu=-\mu+m, \nu=-\nu+n .
$$

so $\mu, \nu$ are either integers or half-integers.

We summarize the result

Lemma 2.3. There are four possible classes of inequivalent real spectral triples over the noncommutative torus, given by the following data. Let $\epsilon_{1}, \epsilon_{2} \in\left\{0, \frac{1}{2}\right\}$. Then $\mathcal{V}_{+}=\mathcal{V}_{-}$is a linear span of the orthonormal vectors $e_{\mu, \nu}$ labelled by $(\mu, \nu) \in\left(\mathbb{Z}+\epsilon_{1}, \mathbb{Z}+\epsilon_{2}\right)$, the grading $\gamma$ being:

$$
\gamma e_{\mu, \nu, \pm}= \pm e_{\mu, \nu, \pm}
$$

the real structure $J$ :

$$
J e_{\mu, \nu, \pm}=e^{ \pm i(\phi \mu+\psi \nu+\theta)} \lambda^{-\mu \nu} e_{-\mu,-\nu, \mp},
$$

and the equivariant hermitian Dirac operator $D$,

$$
D e_{\mu, \nu, \pm}=d_{\mu, \nu}^{ \pm} e_{\mu, \nu, \mp}
$$

which is determined by the order-one condition.

Proof. First let us prove the existence and uniqueness of an equivariant $D$ satisfying the order-one condition. From the fact that $D=D^{\dagger}$ we learn

$$
d_{\mu, \nu}^{+}=\left(d_{\mu, \nu}^{-}\right)^{*}
$$


The order one condition gives the equations:

$$
\begin{aligned}
\left(d_{\mu+1, \nu}^{+}-d_{\mu, \nu}^{+}\right) e^{i \phi} & =e^{-i \phi}\left(d_{\mu, \nu}^{+}-d_{\mu-1, \nu}^{+}\right), \\
\left(d_{\mu+1, \nu}^{+}-d_{\mu, \nu}^{+}\right) e^{i \psi} & =e^{-i \psi}\left(d_{\mu+1, \nu-1}^{+}-d_{\mu, \nu-1}^{+}\right), \\
\left(d_{\mu, \nu+1}^{+}-d_{\mu, \nu}^{+}\right) e^{i \phi} & =e^{-i \phi}\left(d_{\mu-1, \nu+1}^{+}-d_{\mu-1, \nu}^{+}\right), \\
\left(d_{\mu, \nu+1}^{+}-d_{\mu, \nu}^{+}\right) e^{i \psi} & =e^{-i \psi}\left(d_{\mu, \nu}^{+}-d_{\mu, \nu-1}^{+}\right),
\end{aligned}
$$

whose only solutions are:

$$
d_{\mu, \nu}^{+}=\left\{\begin{aligned}
\tau_{0} e^{-2 i \phi \mu-2 i \psi \nu}+\epsilon, & \text { when } \phi \neq 0, \psi \neq 0, \\
\tau_{1} \mu+\tau_{0} e^{-2 i \psi \nu}+\epsilon, & \text { when } \phi=0, \psi \neq 0, \\
\tau_{2} \nu+\tau_{0} e^{-2 i \phi \mu}+\epsilon, & \text { when } \phi \neq 0, \psi=0, \\
\tau_{1} \mu+\tau_{2} \nu+\epsilon . & \text { when } \phi=0, \psi=0,
\end{aligned}\right.
$$

Finally, demanding that $J D=D J$ we have, in each of the possible cases:

- $\phi \neq 0, \psi \neq 0$

$$
-\left(\tau_{0}\right)^{*} e^{i \phi \mu+i \psi \nu}-\epsilon^{*} e^{-i \phi \mu-i \psi \nu}=\left(\tau_{0}\right)^{*} e^{-i \phi \mu-i \psi \nu}+(\epsilon)^{*} e^{i \phi \mu+i \psi \nu},
$$

which holds if $\tau_{0}=-\epsilon$,

- $\phi=0, \psi \neq 0$

$$
-\left(\tau_{1}\right)^{*} \mu-\left(\tau_{0}\right)^{*} e^{i \psi \mu}-\epsilon^{*} e^{-i \psi \mu}=-\left(\tau_{1}\right)^{*} \mu+\tau_{0} e^{-i \psi \mu}+\epsilon e^{i \psi \mu},
$$

which holds for arbitrary $\tau_{1}$ and $\tau_{0}=-\epsilon$,

- $\phi \neq 0, \psi=0$

$$
-\left(\tau_{2}\right)^{*} \nu-\left(\tau_{0}\right)^{*} e^{i \phi \mu}-\epsilon^{*} e^{-i \phi \mu}=-\left(\tau_{2}\right)^{*} \nu+\tau_{0} e^{-i \phi \mu}+\epsilon e^{i \phi \mu},
$$

which holds for arbitrary $\tau_{2}$ and $\tau_{0}=-\epsilon$,

- $\phi \neq 0, \psi \neq 0$

$$
-\left(\tau_{1}\right)^{*} \mu-\left(\tau_{2}\right)^{*} \nu-\epsilon^{*}=-\left(\tau_{1}\right)^{*} \mu+-\left(\tau_{2}\right)^{*} \nu+\epsilon^{*},
$$

which holds for arbitrary $\tau_{1}, \tau_{2}$ and $\epsilon=0$. 
The necessary final condition to fix the spectral data comes either from the requirement of the spectral properties of the Dirac operator or the Hochschild cycle condition. In the first case, it is obvious that only for $\phi=\psi=0$, the growth of eigenvalues of the Dirac operator corresponds to the required axioms and $D$ has compact resolvent. The Hochschild cocycle condition is more complicated, and we prove it next.

Lemma 2.4. The Hochschild cycle condition, that is, that there exists:

$$
c=\sum c_{0} \otimes c_{0}^{o} \otimes c_{1} \otimes c_{2} \in Z_{2}\left(\mathcal{A}, \mathcal{A} \otimes \mathcal{A}^{o}\right)
$$

such that

$$
\gamma=\pi(c)=\pi\left(c_{0}\right)\left(J^{-1} \pi\left(c_{0}^{o}\right) J\right)\left[D, \pi\left(c_{1}\right)\right]\left[D, \pi\left(c_{1}\right)\right],
$$

is satisfied only if $\phi=\psi=0$.

Proof. To prove it, we start with the case $\phi \neq 0, \psi \neq 0$ and observe,

$$
[D, \pi(U)]_{ \pm}=\left(e^{\mp 2 i \phi}-1\right) U D_{ \pm}^{\prime}, \quad[D, \pi(V)]_{ \pm}=\left(e^{\mp 2 i \psi}-1\right) U D_{ \pm}^{\prime}
$$

where the sign denotes the restriction of the operators to $\mathcal{V}_{ \pm}$, and $D^{\prime}=D+\tau_{0}$. Moreover:

$$
D^{\prime} \pi(U)=e^{\mp 2 i \phi} \pi(U) D^{\prime}, \quad D^{\prime} \pi(V)=e^{\mp 2 i \psi} \pi(v) D^{\prime} .
$$

Any expression of the type: $\pi\left(a_{0}\right)\left[D, \pi\left(a_{1}\right)\right]\left[D, \pi\left(a_{2}\right)\right]$, where $a_{0}, a_{1}, a_{2}$ are homogeneous polynomials in $U, V$ (since we are working with the algebra of polynomials, we can always restrict ourselves to this case), must therefore be proportional to:

$$
C\left(a_{1}, a_{2}\right) \pi\left(a_{0}\right) \pi\left(a_{1}\right) \pi\left(a_{2}\right),
$$

where $C\left(a_{1}, a_{2}\right)$ is a complex number depending only on multi-degree of polynomials $a_{1}, a_{2}$ and the $\psi$ and $\phi$.

Now, assuming that the cocycle condition holds, we would have a decomposition of 1 (when restricted to $\mathcal{V}_{+}$) as a finite sum of homogeneous polynomials in $U, V, U^{o}, V^{o}$. This, however, is not possible, unless the polynomials are all of degree 0 . Hence $c_{0}^{o}$ from the cocycle must be 1 .

Assume next that the cocyle $c$ is trivial, i.e. $c=b c^{\prime}$. It can be easily verified that in such case its image $\pi(c)$ is a sum of commutators of the type

$$
\left[\pi\left(a_{0}^{\prime}\right)\left[D, \pi\left(a_{1}^{\prime}\right)\right]\left[D, \pi\left(a_{2}^{\prime}\right)\right], \pi\left(a_{3}^{\prime}\right)\right] .
$$


Using the previous result, we can decompose it into the sum of commutators of homogeneous polynomials and we immediately see that for the algebra of the noncommutative torus commutators cannot give a polynomial of degree 0 . Hence, no trivial cocycle can have $\gamma$ as its image.

On the other hand, using the results on the Hochschild homology of the noncommutative torus [6] we explicitly verify that for the unique nontrivial cocycle (up to multiplication):

$$
c_{0}=U^{*} V^{*} \otimes V \otimes U-V^{*} U^{*} \otimes U \otimes V,
$$

its image, $\pi\left(c_{0}\right)$ vanishes. Therefore, in this case, the Hochschild cocycle condition cannot be satisfied.

In the remaining case ( $\phi=0$ and $\psi \neq 0$, for instance) we use similar arguments. We have:

$$
[D, \pi(U)]_{ \pm}=\tau_{\mu}^{ \pm} U I_{r}, \quad[D, \pi(V)]_{ \pm}=\left(e^{\mp 2 i \psi}-1\right) U D_{ \pm}^{\prime},
$$

where $I_{r} e_{\mu \nu, \pm}=e_{\mu \nu, \mp}$ and $D^{\prime} e_{\mu, n u, \pm}=e^{-2 i \psi \nu} e_{\mu, n u, \mp}$.

Repeating the arguments from previous considerations, we obtain that for any expression of the considered type, it might have three components:

$$
\begin{aligned}
C\left(a_{1}, a_{2}\right) \pi\left(a_{0}\right) \pi\left(a_{1}\right) \pi\left(a_{2}\right) & +C^{\prime}\left(a_{1}, a_{2}\right) \pi\left(a_{0}\right) \pi\left(a_{1}\right) \pi\left(a_{2}\right) I_{r} D^{\prime} \\
& +C^{\prime \prime}\left(a_{1}, a_{2}\right) \pi\left(a_{0}\right) \pi\left(a_{1}\right) \pi\left(a_{2}\right) D^{\prime} I_{r},
\end{aligned}
$$

with three complex coefficients, depending only on the multi-degree of $a_{1}, a_{2}$ and $\psi$.

The difference here is the appearance of $D^{\prime}$, but again, it is sufficient to verify that no such finite sum can be proportional to the identity, when restricted to $\mathcal{V}_{+}$, unless all $C^{\prime}, C^{\prime \prime}$ vanish and the degree of $a_{0} a_{1} a_{2}$ is zero.

We can further follow the same arguments for the commutator presentation of trivial Hochschild cycles, checking again explicitly that for the nontrivial $c_{0}$, its image has non-zero coefficients $C^{\prime}$ and $C^{\prime \prime}$ and for this reason its image cannot be $\gamma$.

For the $\psi=0, \phi=0$ case, we calculate that

$$
\begin{aligned}
\gamma=\frac{1}{\tau_{\mu}^{*} \tau_{\nu}-\tau_{\mu} \tau_{\nu}^{*}} & \left(\pi\left(V^{*}\right) \pi\left(U^{*}\right)[D, \pi(U)][D, \pi(V)]\right. \\
& \left.-\pi\left(U^{*}\right) \pi\left(V^{*}\right)[D, \pi(V)][D, \pi(U)]\right) .
\end{aligned}
$$

provided that $\tau_{\mu} \tau_{\nu}^{*} \neq \tau_{\mu}^{*} \tau_{\nu}$. 
We can now state:

Theorem 2.5. There are four inequivalent equivariant spin structures on the 2dimensional noncommutative torus, with a unique choice of equivariant Dirac operator for each spin structure:

$$
d_{\mu, \nu}^{+}=\tau_{\mu} \mu+\tau_{\nu} \nu
$$

which satisfies the Hochschild cycle condition, provided that $\tau_{\mu} \tau_{\nu}^{*} \neq \tau_{\mu}^{*} \tau_{\nu}$. The spectrum of the equivariant Dirac Operator depends on the spin structure.

Proof. As the previous lemmas showed the construction of the spectral data, we only need to show their inequivalence and the dependence of the spectrum of the Dirac operator on the choice of the class.

In order to see that the different reality structures we found are not equivalent we need to find the same presentation of the spectral geometries. This is achieved by relabelling the indices so that their are all integers.

We obtain, on the Hilbert space with the basis labelled by integers $m, n$ :

\begin{tabular}{|l|l||c|c|}
\hline$\epsilon_{\mu}$ & $\epsilon_{\nu}$ & $J|m, n, \pm\rangle$ & $D|m, n, \pm\rangle$ \\
\hline 0 & 0 & $\pm \lambda^{-m n}|-m,-n, \mp\rangle$ & $\left(\tau_{1} m+\tau_{2} n\right)|-m,-n, \pm\rangle$ \\
\hline 0 & $\frac{1}{2}$ & $\pm \lambda^{-m\left(n \mp \frac{1}{2}\right)}|-m,-n+1, \mp\rangle$ & $\left(\tau_{1} m+\tau_{2} n+\frac{1}{2}\right)|-m,-n, \pm\rangle$ \\
\hline$\frac{1}{2}$ & 0 & $\pm \lambda^{-\left(m \mp \frac{1}{2}\right) n}|-m+1,-n, \mp\rangle$ & $\left(\tau_{1} m+\tau_{2} n+\frac{1}{2}\right)|-m,-n, \pm\rangle$ \\
\hline$\frac{1}{2}$ & $\frac{1}{2}$ & $\pm \lambda^{-\left(m \mp \frac{1}{2}\right)\left(n \mp \frac{1}{2}\right)}|-m+1,-n+1, \mp\rangle$ & $\left(\tau_{1} m+\tau_{2} n+1\right)|-m,-n, \pm\rangle$ \\
\hline
\end{tabular}

So two of the above cases have clearly a different spectrum of the Dirac operator than the other two (for instance, for $\epsilon_{\mu} \neq \epsilon_{\nu}, 0$ is not in the spectrum of $D$ ). It is thus immediately evident that the two pairs corresponding to the different spectra of $D$ are not unitarily equivalent.

Let us prove the mutual inequivalence of the two cases within each pair. We shall show that there does not exist a unitary operator $W$ on $\mathcal{V}$, such that $J_{\epsilon_{\mu}^{\prime} \epsilon_{\nu}^{\prime}}=$ $W J_{\epsilon_{\mu} \epsilon_{\nu}} W^{*}$, and which leaves the remaining data of the spectral triple unchanged, in particular

$$
\begin{aligned}
W \gamma W^{*} & =\gamma \\
W \pi(a) W^{*} & =\pi(a) \quad \forall a \in \mathcal{A}\left(T_{\lambda}\right)
\end{aligned}
$$


From the first of these equations, (32), it follows that $W$ is blockdiagonal, $W$ : $\mathcal{V}_{ \pm} \rightarrow \mathcal{V}_{ \pm}$. To make use of (33) we first observe that $e_{n, m, \pm}=\pi\left(U^{n}\right) \pi\left(V^{m}\right) e_{0,0, \pm}$ for all $n, m$. We shall denote

$$
W e_{0,0, \pm}=\sum_{k, l} w_{k l}^{ \pm} e_{k, l, \pm}
$$

Using (33), we have

$$
\begin{array}{rlr}
W e_{n, m, \pm} & =W \pi\left(U^{n}\right) \pi\left(V^{m}\right) e_{0,0, \pm}=\pi\left(U^{n}\right) \pi\left(V^{m}\right) W e_{0,0, \pm} \\
& = & \pi\left(U^{n}\right) \pi\left(V^{m}\right)\left(\sum_{k, l} w_{k l}^{ \pm} e_{k, l, \pm}\right) \\
& = & \sum_{k, l} \lambda^{-m k} w_{k l}^{ \pm} e_{k+n, l+m, \pm} .
\end{array}
$$

Thus $W$ is completely determined by the coefficients $w_{k l}^{ \pm}$. The requirement $W J_{\epsilon_{\mu} \epsilon_{\nu}} W^{*}=$ $J_{\epsilon_{\mu}^{\prime} \epsilon_{\nu}^{\prime}}$, gives the following equation:

$$
w_{-k,-l}^{ \pm} j_{\epsilon_{\mu}, \epsilon_{\nu}, \pm}(n+k, m+l)=\lambda^{2 m k} w_{k, l}^{\mp} j_{\epsilon_{\mu}^{\prime}, \epsilon_{\nu}^{\prime}, \pm}(n, m), \quad \forall n, m, k, l \in \mathbb{Z},
$$

where

$$
J_{\iota} e_{n, m, \pm}=j_{\iota}(n, m) e_{-n,-m, \mp}, \quad \iota=\left\{\left(\epsilon_{\mu} \epsilon_{\nu}\right),\left(\epsilon_{\mu}^{\prime} \epsilon_{\nu}^{\prime}\right)\right\} .
$$

Taking the $j_{\iota}(n, m)$ from the table above and inserting them into the equation for the $w_{k l}^{ \pm}$one easily sees that there only exists a solution if $J_{\epsilon_{\mu}^{\prime}, \epsilon_{\nu}^{\prime}}=J_{\epsilon_{\mu}, \epsilon_{\nu}}$ in which case $W= \pm \mathrm{id}$. Thus there does not exist a unitary that intertwines distinct reality structures $J_{\epsilon_{\mu} \epsilon_{\nu}}$.

Finally, it is interesting to note that, were $W$ not required to commute with $\gamma$ and the algebra representation, there would exist such a unitary. For example, an unitary $W$ which intertwines $J_{00}$ and $J_{0 \frac{1}{2}}$,

$$
J_{0 \frac{1}{2}}=W^{*} J_{00} W
$$

is given by:

$$
W e_{m, n,+}=\lambda^{\frac{1}{2} m} e_{m, n-1,-}, \quad W e_{m, n,-}=e_{m, n,+} \cdot
$$

Indeed, then

$$
\begin{aligned}
W^{*} J_{00} W e_{m, n,+} & =W^{*} J_{00} \lambda^{\frac{1}{2} m} e_{m, n-1,-}= \\
& =W^{*} \lambda^{-m(n-1)} \lambda^{-\frac{1}{2} m} e_{-m,-n+1,+} \\
& =\lambda^{-m\left(n-\frac{1}{2}\right)} e_{-m,-n+1,-},
\end{aligned}
$$


and

$$
\begin{aligned}
W^{*} J_{00} W e_{m, n,-} & =W^{*} J_{00} e_{m, n,+}=W^{*} \lambda^{-m n} e_{-m,-n,-} \\
& =\lambda^{-\frac{1}{2} m} \lambda^{-m n} e_{-m,-n+1,+} \\
& =\lambda^{-m\left(n+\frac{1}{2}\right)} e_{-m,-n+1,+}
\end{aligned}
$$

\section{Conclusions}

We have shown that the noncommutative torus has, similarly as in the classical $\lambda=1$ case, four inequivalent spin structures. It is not surprising that the spin structures are closely related to the reality structure $J$. It is quite instructive, however, that the pure algebraic conditions for $J$ and the Dirac operator are not sufficient and one needs either the Hochschild cocycle condition or the restriction due to the spectral properties of the Dirac operator. Note that in [4] this was also shown to rule out spin bundles with the wrong topology over the commutative sphere $S^{2}$, leaving precisely one (real) Spin structure in that case.

The "nonexisting" spurious classes of the reality operator $J$, which do not lead to true Dirac operators have no classical (commutative) counterpart. For this reason, it is hard to compare the construction with the steps of Connes' reconstruction theorem for spin geometries [3] in order to see whether their existence is a shadow of some other structures.

\section{References}

[1] Ch. Bär, "Dependence of the Dirac spectrum on the spin structure" in: Sminaires et Congrs 4, Global Analysis and Harmonic Analysis, J.P. Bourguignon, T. Branson, O. Hijazi (eds.), 2000, 17-33,

[2] A. Connes, "Noncommutative geometry and reality", J. Math. Phys. 36 (1995), 6194-6231.

[3] J. M. Gracia-Bondía, J. C. Várilly and H. Figueroa, Elements of Noncommutative Geometry, Birkhäuser, Boston, 2001.

[4] M. Paschke, "Von Nichtkommutativen Geometrien, ihren Symmetrien, und etwas Hochenergiephysik", Ph.D. thesis, Mainz 2001 
[5] A. Sitarz, "Equivariant spectral triples", in Noncommutative Geometry and Quantum Groups, P. M. Hajac and W. Pusz, eds. Banach Centre Publications 61, IMPAN, Warszawa, 2003; pp. 231-263.

[6] M. Wambst, "Hochschild and cyclic homology of the quantum multiparametric torus", Journal of Pure and Applied Algebra 114 (1997) 321-329. 